\title{
DISTRIBUTIVE PROPERTIES OF SET OPERATORS
}

\section{WILLIAM WERNICK}

A set operator, designated by small letters, $a, b, \cdots$, is one which takes subsets $A, B, \cdots$ of a given space $S$ into subsets $a A, a B, \cdots$ $C S$. A property of a set operator $a$ is a constant relation between argument and image sets under $a$ and is expressed in a statement of equation or inclusion, for example, $a(A+B)=a A \cdot a B$ or $A \subset b A$ where $a, b$ may mean, for example, "complement" or "closure."

We investigate properties expressed by relations of the form:

$$
a\left(A f_{1} B\right) \cdot R: a A f_{2} a B
$$

where $f_{1}, f_{2}$ are either set sum: + , or set product: $\cdot$, and where $R$ is either $=, \supset$, or $C$. A property defined by such a relation (1) is a distributive property, but not all distributive properties can be defined by (1), for example $a(A+B)=A \cdot a B+B \cdot a A$, and so on.

When $f_{1}, f_{2}, R$ are given constant values, (1) becomes the statement of a specific distributive property of $a$. We now list them individually for reference. Properties of monotonicity and inverse-monotonicity ( $\alpha_{13}$ and $\alpha_{14}$ below) are closely related to properties of distributivity so they are listed in the table also. (The arrow, $\rightarrow$, is used for implication throughout this paper.)

TABLE I

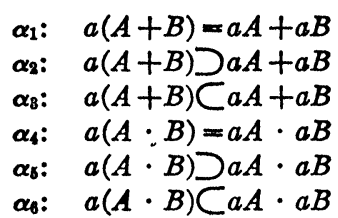

$\begin{array}{rr}\alpha_{7}: & a(A+B)=a A \cdot a B \\ \alpha_{8}: & a(A+B) \supseteq a A \cdot a B \\ \alpha_{9}: & a(A+B) \subset a A \cdot a B \\ \alpha_{10}: & a(A \cdot B)=a A+a B \\ \alpha_{11}: & a(A \cdot B) \supseteq a A+a B \\ \alpha_{12}: & a(A \cdot B) \subset a A+a B \\ A \subset B \rightarrow a A C_{B B} & \\ A C_{B \rightarrow a A \supset a B} & \end{array}$

To say that $a$ has property $\alpha_{1}$ (notation $a: \alpha_{1}$ ) means: "For every $A, B, a(A+B)=a A+a B$." These properties $\alpha_{i}$ are obviously not independent, for example, $a: \alpha_{1} \rightarrow a: \alpha_{2}, \alpha_{3}$ (which we may shorten, at our convenience, to $\alpha_{1} \rightarrow \alpha_{2}, \alpha_{3}$ ).

Our first main question is: if we hypothesize to $a$ a single property $\alpha_{i}$, what other properties must $a$ have? This is completely answered by the following diagram of implications:

Presented to the Society, October 26, 1940; received by the editors July 1.8, 1944. 


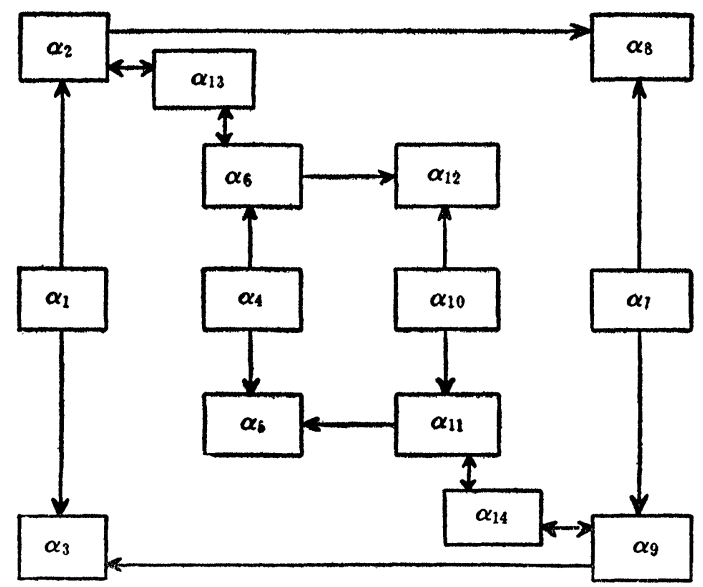

Since most of these implications are quite obvious, I present only two proofs which are models for the not too obvious implications. Introduction of the hypothesis is indicated by subscript $h$.

$$
\begin{aligned}
& \alpha_{2} \rightarrow \alpha_{13}: A \subset B \rightarrow B=A+B \rightarrow a B=a(A+B) \supset_{h} a A+a B \\
& \supset a A \rightarrow a A \subset a B \\
& \alpha_{14} \rightarrow \alpha_{11}: \quad\left[A \cdot B \subset A \rightarrow a(A \cdot B) \supset_{h} a A ;\right. \\
& \left.A \cdot B \subset B \rightarrow a(A \cdot B) \supset_{h} a B\right] \rightarrow a(A \cdot B) \supset a A+a B .
\end{aligned}
$$

Sometimes we know or wish to assume that a given operator $a$ does not have a specific property $\alpha_{i}$ (notation $\overline{a: \alpha_{i}}$ ) and we wish to know the implications of this.

We define the property $\bar{\alpha}_{i}$ thus: $a: \bar{\alpha}_{i}$ if and only if $\overline{a: \alpha_{i}}$; and easily get the implications for $\bar{\alpha}_{i}$ from diagram I by replacing $\alpha_{i}$ by $\bar{\alpha}_{i}$ and reversing every arrow. The resulting diagram, which will not be drawn, can be called II.

Our second main question is: if $a: \alpha_{i}$ what other properties may be hypothesized for $a$ (from now on, unless otherwise noted, "property," and " $\alpha_{i}$ " will include the properties $\bar{\alpha}_{i}$ ).

The distributive character of an operator $a$ is (said to be) determined with respect to $\alpha_{i}$ (notation $a: i$ ) if it is known definitely that $a: \alpha_{i}$ or that $a: \bar{\alpha}_{i}$. Otherwise the distributive character of $a$ is not determined with respect to $\alpha_{i}$ (notation $a: \bar{i}$ ) and we may then hypothesize $a: \alpha_{i}$ (or $\left.a: \bar{\alpha}_{i}\right)$, provided there is no subsequent contradiction between 
properties already hypothesized for $a$ and the consequences of the joint hypothesis of $\alpha_{i}$ (or $\bar{\alpha}_{i}$ ) and these properties. ${ }^{1}$

All the implications from the joint hypothesis of any of the $\alpha_{i}$ are derivable from the implication diagrams I and II, already given, and III, which is indicated below:

III

$$
\alpha_{2}, \alpha_{3} \rightarrow \alpha_{1} ; \quad \alpha_{5}, \alpha_{6} \rightarrow \alpha_{4} ; \quad \alpha_{8}, \alpha_{9} \rightarrow \alpha_{7} ; \quad \alpha_{11}, \alpha_{12} \rightarrow \alpha_{10} .
$$

These implications are immediate consequences of the definitions in Table I and the usual meanings for set sum, product, and inclusion.

If $a: \alpha_{i}$, then from I and II we can easily determine all the $j$ for which $a: \bar{j}$. If $a: \alpha_{i}$ and $a: j$ then we test the self-consistency of $a: \alpha_{i}, \alpha_{j}$, and of $a: \alpha_{i}, \bar{\alpha}_{j}$, using I, II and III. If both of these are possible (that is, self-consistent) then $\alpha_{j}$ is independent of $\alpha_{i}$, If, furthermore, $a: \bar{\alpha}_{i}, \alpha_{j}$ and $a: \bar{\alpha}_{i}, \bar{\alpha}_{j}$ are also possible, then $\alpha_{i}$ and $\alpha_{j}$ are completely independent of each other. We investigate the complete existential theory of all the $\alpha_{i}$, following the procedure outlined above, so that if $a: \alpha_{i}, \alpha_{j}, \vec{k}$, we shall investigate $a: \alpha_{i}, \alpha_{j}, \alpha_{k}$, and $a: \alpha_{i}, \alpha_{j}, \bar{\alpha}_{k}$; and so on.

If, finally, $a: i$ for $i=1, \cdots, 14$, then we say that the distributive character of $a$ is completely determined. It is easily seen that the distributive character of an operator can be completely determined in a variety of ways, for example, if $a: \alpha_{1}, \alpha_{5}$, then $a: i$ for every $i$; likewise if $a: \alpha_{8}, \alpha_{10}$. The selection of properties that completely determine the distributive character of an operator can be said to define a particular distributive type, thus two operators of the same type possess exactly the same selection of properties $\left\{\alpha_{i}\right\}$. The complete existential theory of these properties as well as all questions on their interdependence will be determined as soon as we find all possible distributive types. ${ }^{2}$

1 The logical distinction between " $\left(a: \alpha_{i}\right)$ or $\left(a: \bar{\alpha}_{i}\right)$ " and " $a:\left(\alpha_{i}\right.$ or $\left.\bar{\alpha}_{i}\right)$ " should be noted. To determine the truth of either "It is now snowing at the North Pole" or "It is now not snowing at the North Pole" would require considerable effort whereas I already know that "It is now either snowing or not snowing at the North Pole."

2 I have made an attempt to list all distinct partially determined (p.d.) operators. With the assumption of one $\alpha_{i}$, there are 20 different p.d. operators. With the assumption of two independent $\alpha_{i}$, there are 5 completely determined (c.d.)and 99 p.d. operators. If we assume three independent $\alpha_{i}$, there are 4 more c.d. and 185 more p.d. operators. If we assume four independent $\alpha_{i}$, we know, from Table II below, that there are 8 more c.d. operators, but I have not determined the number of p.d. operators with 4 or more independent $\alpha_{i}$ assumed. The enumeration is simple and cumulative but tedious and not especially rewarding. The total number of p.d. operators is probably less than 500 , but I see no importance in their exact number or constituency so they are not listed here. 
The distributive types have been determined, and they are exhibited in Table II below. Each line represents a single type $T_{i}$. A " + " in row $i$, column $j$ indicates that an operator of type $T_{i}$ is assumed to have property $\alpha_{j}$; a " - " indicates that the operator is asasumed to have property $\bar{\alpha}_{j}$.

TABLE II

\begin{tabular}{|c|c|c|c|c|c|c|c|c|c|c|c|c|c|c|c|c|}
\hline & $\alpha_{1}$ & $\alpha_{2}$ & $\alpha_{3}$ & $\alpha_{4}$ & $\alpha_{5}$ & $\alpha_{6}$ & $\alpha_{7}$ & $\alpha_{8}$ & $\alpha_{9}$ & $\alpha_{10}$ & $\alpha_{11}$ & $\alpha_{12}$ & $\alpha_{18}$ & $\alpha_{14}$ & Defining Sets & \\
\hline$T_{1}$ & & + & + & + & + & + & + & + & + & + & + & + & + & + & & \\
\hline$r_{2}$ & + & + & $t$ & + & + & + & - & + & - & - & - & + & + & - & & 1) \\
\hline$T_{3}$ & + & + & + & - & - & + & - & + & - & - & - & + & + & - & & 1) \\
\hline$T_{4}$ & - & + & - & + & $t$ & + & - & + & - & - & - & + & + & - & & \\
\hline$T_{5}$ & - & + & - & - & - & + & - & + & - & - & - & + & + & - & & 3) \\
\hline$T_{6}$ & - & - & + & - & + & - & + & + & + & + & + & + & - & + & & 3) \\
\hline$T_{7}$ & - & - & + & - & $t$ & - & + & + & $t$ & - & + & - & - & + & & \\
\hline$T_{8}$ & - & - & + & - & $t$ & - & - & - & $t$ & + & $t$ & + & - & + & & \\
\hline$T_{9}$ & - & - & 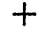 & - & + & - & - & - & 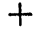 & - & t & - & - & + & & 3) \\
\hline$T_{10}$ & - & - & - & - & + & - & - & + & - & - & - & t & - & - & & (9) \\
\hline$T_{1}$ & - & - & - & - & + & - & - & + & - & - & - & - & - & - & & 3) \\
\hline$T_{1}$ & - & - & - & - & + & - & - & - & - & - & - & + & - & - & & (3) \\
\hline$T_{1}$ & - & - & + & - & + & - & - & - & - & - & - & - & - & - & & (3) \\
\hline$\Gamma_{1}$ & - & - & + & - & - & - & - & + & - & - & - & + & - & - & & (3) \\
\hline$T_{1}$ & - & - & + & - & - & - & - & + & - & - & - & - & - & - & 3 & (3) \\
\hline$T_{1}$ & - & - & + & - & - & - & - & - & - & - & - & + & - & - & 3, & (1) \\
\hline$T_{1}$ & - & - & + & - & - & - & - & - & - & - & - & - & - & - & 3 , & (1) \\
\hline$T_{1}$ & - & - & - & - & + & - & - & + & - & - & - & + & - & - & $\mathbf{2}$, & (3) \\
\hline$T_{19}$ & - & - & - & - & + & - & - & + & - & - & - & - & - & - & $\overline{3}$ & (1) \\
\hline$T_{2}$ & - & - & - & - & + & - & - & - & - & - & - & + & - & - & & (1) \\
\hline$T_{2}$ & - & - & - & - & + & - & - & - & - & - & - & - & - & - & $\overline{3}$ & (1) \\
\hline$T_{2}$ & - & - & - & - & - & - & - & + & - & - & - & + & - & - & & (3) \\
\hline$T_{28}$ & - & - & - & - & - & - & - & + & - & - & - & - & - & - & & (1) \\
\hline$T_{24}$ & - & - & - & - & - & - & - & - & - & - & - & + & - & - & & (1) \\
\hline$T$ & - & - & - & - & - & - & - & - & - & - & - & - & - & - & $\overline{3}, \overline{5}, \overline{8}, \overline{12}$ & (1) \\
\hline
\end{tabular}

Questions of redundancy, though not essential here, have a certain esthetic appeal, and also a later practical use. It is always possible, for each $T_{i}$, to make a selection of properties from among the 14 on that line, which have the following 2 properties collectively:

1. Sufficiency. All the other properties on that line can be deduced from those of this selection (that is, set) of properties.

2. Non-redundancy. No property of this set can be deduced from the other properties of this set.

Such a set will be called a "defining set" of properties for that type. All defining sets for each type have been determined. In the last column of Table II, a defining set is given for each type, and, in 
parentheses, the total number of such sets for that type. (For purposes of brevity, in this column only, we have replaced $\alpha_{i}$ by $i$ ). The equivalence (as shown in diagram I) of $\alpha_{2}, \alpha_{13}, \alpha_{6}$ or of $\alpha_{9}, \alpha_{14}, \alpha_{11}$ is a frequent reason for the multiplicity of defining sets for a particular type.

From Table II we can quickly determine whether or not two properties are completely independent; for example, $\alpha_{7}$ and $\alpha_{10}$, both of which are enjoyed by the operator "complement," are completely independent, since, from $T_{6}, T_{7}, \alpha_{10}$ is independent of $\alpha_{7}$, and from $T_{6}, T_{8}, \alpha_{7}$ is independent of $\alpha_{10}$. Similarly we could determine the complete independence for groups of properties.

We may show that a given set is a defining set by proving suffciency and non-redundancy; for example, for $T_{7}$ we test $\left\{\alpha_{7}, \bar{\alpha}_{12}\right\}$. From I, $\alpha_{7} \rightarrow \alpha_{8}, \alpha_{9}, \alpha_{3}, \alpha_{14}, \alpha_{11}, \alpha_{5}$; from II, $\bar{\alpha}_{12} \rightarrow \bar{\alpha}_{13}, \bar{\alpha}_{6}, \bar{\alpha}_{4}, \bar{\alpha}_{13}, \bar{\alpha}_{2}, \bar{\alpha}_{1}$; therefore this set is sufficient. From Table II there exist types $T_{6}, T_{7}$, $T_{8}, T_{9}$, which show the complete independence of $\alpha_{7}, \alpha_{12}$, therefore this set is non-redundant and thus a defining set.

Our second main question: "If $a: \alpha_{i}$ what other properties may $a$ have?" is obviously answered in Table II.

We shall not prove here that these are the only possible types (which could be done from our definitions) but shall show by examples that functions exist for each of the 25 types. We use mainly a Boolean algebra of four elements (for $T_{13}$ and $T_{22}$ we require eight elements) in which 1 is the universal, 0 is the null, and 2, 3 are the mutually exclusive, exhaustive elements. The tables for sum, product and inclusion are indicated below:

\begin{tabular}{l|llll}
+ & 1 & 2 & 3 & 0 \\
\hline 1 & 1 & 1 & 1 & 1 \\
2 & 1 & 2 & 1 & 2 \\
3 & 1 & 1 & 3 & 3 \\
0 & 1 & 2 & 3 & 0
\end{tabular}

\begin{tabular}{l|llll}
$\cdot$ & 1 & 2 & 3 & 0 \\
\hline 1 & 1 & 2 & 3 & 0 \\
2 & 2 & 2 & 0 & 0 \\
3 & 3 & 0 & 3 & 0 \\
0 & 0 & 0 & 0 & 0
\end{tabular}

\begin{tabular}{l|llll}
$C$ & 1 & 2 & 3 & 0 \\
\hline 1 & $Y$ & $N$ & $N$ & $N$ \\
2 & $Y$ & $Y$ & $N$ & $N$ \\
3 & $Y$ & $N$ & $Y$ & $N$ \\
0 & $Y$ & $Y$ & $Y$ & $Y$
\end{tabular}

An operator for each type will be given by listing the values for $a(1), a(2), a(3), a(0)$ in that order. Thus, from the table below, an example of an operator of type $T_{1}$ is the operator for which $a(1)=1$ $a(2)=1, a(3)=1, a(0)=1$, and so on. ${ }^{3}$

${ }^{3}$ The simplest way to check these is to use the defining sets given in Table II, since if an operator has the properties required in the defining set of $T_{i}$, then it must, from the definition of "defining set," have all the other properties required for an operator of type $T_{i}$. 


$$
\begin{aligned}
& T_{1}:(1,1,1,1) \\
& T_{2}:(1,2,3,0) \\
& T_{3}:(1,1,1,0) \\
& T_{4}:(1,0,0,0) \\
& T_{5}:(1,2,2,0) \\
& T_{6}:(0,3,2,1) \\
& T_{7}:(0,0,0,1) \\
& T_{8}:(0,1,1,1) \\
& T_{9}:(0,2,2,1) \\
& T_{10}:(2,1,0,0) \\
& T_{11}:(2,2,0,1) \\
& T_{12}:(2,1,3,1) \\
& T_{13}: \text { given below }
\end{aligned}
$$$$
T_{14}:(2,2,1,3)
$$$$
T_{15}:(2,2,2,3)
$$$$
T_{16}:(0,1,2,0)
$$$$
T_{17}:(0,3,3,2)
$$$$
T_{18}:(1,0,2,2)
$$$$
T_{19}:(1,0,0,1)
$$$$
T_{20}:(2,3,3,3)
$$$$
T_{21}:(2,3,3,1)
$$$$
T_{22} \text { : given below }
$$$$
T_{23}:(1,3,3,2)
$$$$
T_{24}:(3,2,2,0)
$$$$
T_{25}:(3,2,2,3)
$$

The 4-element algebra is provably inadequate for an example of $T_{13}$ and $T_{22}$, so we use 8 elements, $1,2,3,4,5,6,7,0$, where 1 is the universal, 0 is the null element, $2,3,4$ are the mutually exclusive and exhaustive elements, and $5=2+3,6=2+4,7=3+4$. The tables for ,$+ \cdot$, and $\subset$ for the algebra can be filled in very easily from the above description, so they will not be given. The operator definitions are now given as before by listing, in order, the value of $a(1), a(2)$, $\cdots, a(0)$.

$$
T_{13}:(2,2,3,4,5,6,7,7) ; \quad T_{22}:(7,2,3,2,3,6,7,2)
$$

Proofs that these operators have the properties listed for their respective types will not be given since they are lengthy and present no special difficulties. ${ }^{4}$

\section{New York City}

4 See previous footnote for easiest method of proof. 